\title{
Detection of selection signatures based on the integrated haplotype score in Chinese Jinnan cattle
}

\author{
Yuanqing Zhang, Dong Chang He, Jing Cheng, Fang Xu, Bo Li, Guang Jin, Xi Zhong Zhang \\ Institute of Animal Husbandry and Veterinary Science, Shanxi Academy of Agricultural Science, Taiyuan 030032, P. R. China
}

\section{A B S T R A C T}

\begin{abstract}
Jinnan cattle, one of the five major breeds of Chinese yellow cattle, are the traditional draft and beef breed in southern Shanxi province, China. Our study aimed to search genes that will allow the selection of important economic traits through selection signature detection, which can help in discovering the action mechanism of positive selection in the variety breeding process of Jinnan cattle.
\end{abstract}

Keywords: Breeding; Integrated haplotype score; Jinnan cattle; Selection signature

\section{BACKGROUND}

Both natural and artificial selection have resulted in cattle breeds that are specialized for particular uses. Several specific genomic regions have been under selection pressure, and it is widely accepted that these genomic sequences, or selection signatures, control or affect key phenotypes that are involved in some traits. Positive selection pressure can reduce or even eliminate the frequency of negative alleles in the offspring's genome(Biswas and Akey, 2006). Thus, continuous selection pressure over a few generations may result in linkage disequilibrium (LD) patterns between mutations and neighboring loci (Sabeti et al., 2002). Therefore, selection signatures in a population could provide genomic information to facilitate selection and provide insights into evolutionary history (Akey et al., 2002; Willing et al., 2010).

The selection signatures are detected by different methods, such as McDonald and Kreitman test (McDonald and Kreitman, 1991), H-statistic (Fay and Wu, 2000), integrated haplotype score (iHS) (Voight et al., 2006a), and extended haplotype homozygosity (EHH) test(Sabeti et al., 2002)). The study found that the EHH test can detect signatures of positive selection in a population between the allele frequency and LD patterns with neighboring alleles (Barendse et al., 2009). EHH is defined as the probability that two randomly chosen chromosomes carrying the aim core haplotype are identical by descent for the entire interval between a certain locus and the core region. As an extension of the EHH, iHS compares EHH between ancestral and derived alleles within a population. Thus, iHS test can avoid the influence of heterogeneous recombination rates across the genome, and the iHS method performs best when a selected allele segregates at intermediate frequencies in the population.

Recently, several selection signatures have been detected in various independent cattle populations or breeds (Pan et al., 2013; Qanbari et al., 2011; Qanbari et al., 2010; $\mathrm{Xu}$ et al., 2015). Chinese Jinnan cattle, an independent population of the major Chinese yellow cattle, is a traditional draft and beef breed in southern Shanxi province, China (An et al., 2005). In this study, we detected iHS selection signatures and explored their potential functional genes in Chinese Jinnan breeds using high-density single nucleotide polymorphism (SNP) genotyping data.

Jinnan is one of the five excellent local cattle breeds in China. Jinnan cattle show the better characteristics such as stronger physique, muscular, heat resistance, and crude feed tolerance. With the transformation of the agricultural industry structure and the introduction of foreign cattle breed, Jinnan cattle faced a severe challenge. The number of Jinnan cattle sharply declined, and the frequency of

\footnotetext{
${ }^{*}$ Corresponding author:

Jing Cheng, Institute of Animal Husbandry and Veterinary Science, Shanxi Academy of Agricultural Science, Taiyuan 030032, P. R. China.

Phone/Fax: (+86 351) 7561076, E-mail: chengjing1104@163.com
} 
purebreds decreased. This study elucidated the selection mechanisms and breeding value of the Jinnan breed.

\section{MATERIALS AND METHODS}

\section{Animals and genotypes}

All cattle used in this study were healthy juvenile Jinnan cattle weighted $300 \pm 30 \mathrm{~kg}$, obtained from Jinnan cattle elite reservation farm. The animals were raised in compliance with the International Guiding Principles for Biomedical Research Involving Animals (http://www. cioms.ch/frame.1985.texts.of.guidelines.htm), housed under room temperature with good ventilation hygienic conditions, and fed ad libitum.

The study population comprised 65 Chinese Jinnan cattle with no genetic relationships. Ear tissues were sampled and snapped frozen in liquid nitrogen for further use. Total DNA was isolated from ear tissues using DNAiso Reagent (Japan, Dalian, China) according to the manufacturer's protocol.

An Illumina Bovine SNP50 bead chip assay (San Diego, CA, USA) and Genome Studio were used for genotyping. PLINK was employed for quality control, where only SNPs located on autosomal chromosomes in the UMD3.1 genome were selected for autozygosity determination, and marker scores with a minor allele frequency of $<5 \%$ were analyzed. After quality control, 39,814 SNPs from 48 individuals had a genotype call rate of $\geq 95 \%$.

\section{iHS test}

The iHS method depends on information regarding the status of the ancestral and derived alleles for each SNP. The ancestral alleles of all bovine SNPs were established before computing iHS (Rocha et al., 2014) (http:/ / genome. jouy.inra.fr/downloads/Bovine_Ancestral_Allele/). The R package 'rehh' was used to compute iHS for each autosomal SNP (Gautier and Vitalis, 2012). Single-site iHS values were computed across the genome for each breed and averaged within nonoverlapping windows of $500 \mathrm{~kb}$. The window size employed was based on the extent of $\mathrm{LD}$, as described previously (Gautier and Naves, 2011). Standardized iHS was calculated as follows:

i HS $=\ln \left(\frac{\text { i H H A }}{\text { i H D }}\right)$

where iHHA and iHHD represent the integrated EHH scores for the ancestral and derived core alleles, respectively. The iHS values were standardized to ensure that they followed a standard normal distribution (Voight et al., 2006b).

\section{Bioinformatics analyses}

Gene annotation was performed by exploring the UMD3.1 locations of genes via NCBI database (fttp://ftp.ncbi. nih.gov/genomes/Bos_taurus/mapview/seq_gene. md.gz). BioMart (www.ensembl.org/biomart) was used to determine the orthologous human gene ID of each gene detected. Enrichment analysis of these genes was performed using DAVID 6.7 by aligning the detected genes with human genes (Huang da et al., 2009). Functional annotations, i.e., Gene Ontology (GO) biological process, GO cellular component, and GO molecular function, were assigned to genes using the functional annotation tool.

\section{RESULTS}

\section{iHS tests}

The selection signatures in Jinnan cattle were detected on the basis of 39,814 SNPs that covered the bovine UMD3.1 genome, with a mean distance of $6.28 \mathrm{~kb}$ between adjacent SNPs. The mean distance between adjacent SNPs per chromosome ranged from $5.74 \mathrm{~kb}$ on chromosome 25 to $7.36 \mathrm{~kb}$ on chromosome 5 . The iHS method identified 19 significant regions in the genome of Chinese Jinnan cattle (Table 1). Genome-wide distribution of the iHS values was used to visualize the chromosomal distribution of selection signatures (Fig. 1). Throughout the entire genome, the lowest iHS, -5.88 , was in chromosome 13 and the highest, 4.84, was in chromosome 4.

\section{Annotation of candidate regions}

Annotation of genomic regions containing selection signatures detected several candidate genes, and those in

Table 1: Genomic regions with significant integrated haplotype scores (iHS) in Chinese Jinnan breeds

\begin{tabular}{lc}
\hline Genomic region $\mathbf{( k b )}$ & Mean|iHS|value \\
\hline Chr1: $73500001-74000000$ & 2.24 \\
Chr1: $9700001-10100000$ & 2.04 \\
Chr5: $21500001-22000000$ & 2.01 \\
Chr5: $56000001-56500000$ & 2.56 \\
Chr7: $56500001-57000000$ & 2.01 \\
Chr8: $31500001-32000000$ & 2.05 \\
Chr8: $37000001-37500000$ & 2.13 \\
Chr8: $40500001-41000000$ & 2.14 \\
Chr13: $48500001-49000000$ & 2.11 \\
Chr14: $20000001-20500000$ & 2.06 \\
Chr14: $21000001-21500000$ & 2.33 \\
Chr14: $21500001-22000000$ & 2.12 \\
Chr14: $22500001-23000000$ & 2.83 \\
Chr14: $23000001-23500000$ & 2.31 \\
Chr14: $26000001-26500000$ & 2.07 \\
Chr14: $27500001-28000000$ & 2.16 \\
Chr14: $28000001-28500000$ & 2.11 \\
Chr14: $29500001-30000000$ & 2.28 \\
Chr27: $1000001-1500000$ & 2.03 \\
\hline
\end{tabular}




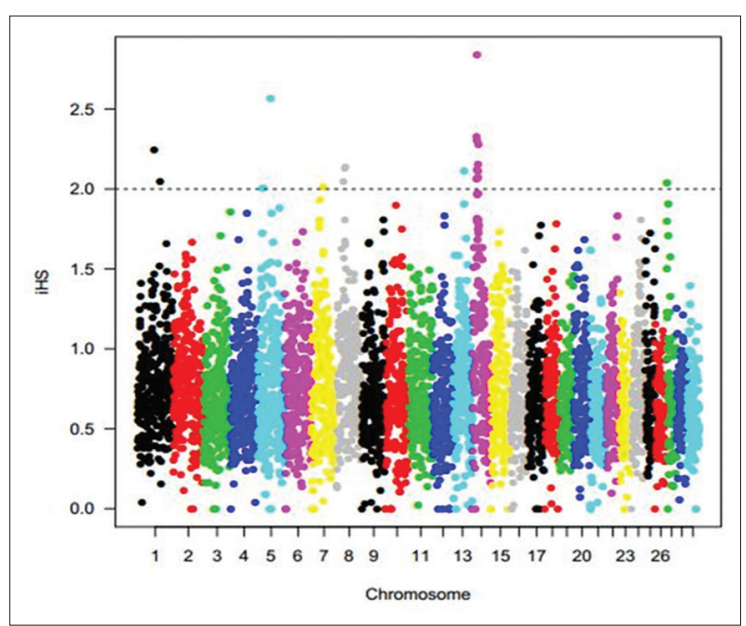

Fig 1. Average integrated haplotype score for each 500-KB window.

significant iHS genomic regions in Jinnan cattle comprised 61 candidate genes (Additional file 1). Moreover, some of our candidate genes were previously detected on the basis of quantitative trait loci (QTL) and genome-wide association study (GWAS) analyses of cattle populations.

\section{Bioinformatics analysis}

To clarify the molecular functions of these genes, we examined their GO classifications. The candidate genes detected in Jinnan cattle were enriched in several significant GO terms (Additional file 2), particularly the HES1, CDK4, and GLI1 genes.

\section{DISCUSSION}

iHS tests for several commercial dairy and beef breeds, including Angus, Belgian Blue, Charolais, Hereford, Holstein-Friesian, Limousin, and Simmental cattle, have identified more than 80 significant selection signature regions (Zhao et al., 2015). The iHS tests for Pinzgau cattle, which is a local breed of domestic cattle from the Pinzgau region of the federal state of Salzburg in Austria, showed that only seven regions had an iHS of $>1.7$ (Kasarda et al., 2015). This suggests that these commercial breeds have undergone stronger and more intensive selection pressure than Chinese Jinnan breed.

Several genes have also been reported on the basis of iHS tests of other commercial dairy and beef breeds (Qanbari et al., 2011; Zhao et al., 2015), such as carbonic anhydrase VIII (CA8) and Ras-related protein Rab-2A (RAB2A) in both Limousin and Charolais; protein-L-isoaspartate (D-aspartate) O-methyltransferase domain containing 1 (PCMTD1), family with sequence similarity 110: member $\mathrm{B}$, UBX domain protein $2 \mathrm{~B}$, cytochrome P450: family 7 : subfamily A: polypeptide 1, and syndecan binding protein in Limousin; and minichromosome maintenance complex component 8 , cardiolipin synthase 1 , and ferritin family member 1 in Holstein-Friesian, which is a dairy breed. None of these genes overlapped with the candidate genes identified in the Angus, Belgian Blue, Hereford or Simmental breeds using iHS tests.

In previous studies, several of the genes identified by the iHS tests were suggested to be associated with health, meat, milk production, and reproductive traits (Additional file 3). Interestingly, the results showed that 47 of 61 candidate genes located in QTL regions were associated with meat and carcass traits, and 11 of 61 candidate genes were reported to be associated with meat and carcass traits on the basis of GWAS data. All these 11 genes were present in the 47 candidate genes in QTL regions associated with meat and carcass traits, including chromosome 8 open reading frame 22, CA8, gamma-glutamyl hydrolase, neuropeptides $\mathrm{B} / \mathrm{W}$ receptor 1 , opioid receptor, kappa 1, PCMTD1, RAB2A, RB1-inducible coiled-coil 1, tocopherol (alpha) transfer protein, leucine rich adaptor protein 1-like, and tyrosinase-related protein-1(Doran et al., 2014; McClure et al., 2012; Nalaila et al., 2012; Nishimura et al., 2012; Saatchi et al., 2014; Veneroni-Gouveia et al., 2012). These results suggested that the Jinnan breed has high genetic potential for beef cattle breeding.

People have documented the association of these genes with the regulation of muscle cell proliferation on the basis of in vitro experiments (Korzelius et al., 2011; Lazaro et al., 2002; Liu et al., 2014; von Baer et al., 2014; von Grabowiecki et al., 2015). Thus, these results provide further evidence that the Jinnan breed is an important resource with unique features for beef cattle breeding.

\section{CONCLUSIONS}

A genome-wide scan based on iHS statistics identified several regions that have been affected by selection in Chinese Jinnan cattle. Among 39,814 SNPs, we estimated positive selection signatures using the iHS test, which showed that the breeding history of Chinese Jinnan cattle had been subject to low positive selection compared with that undergone by commercial dairy and beef breeds. Annotation of the genome regions with selection signatures detected 61 candidate genes, including 11 interesting genes annotated in significant regions that overlapped with both QTL and GWAS data associated with meat traits in cattle populations. GO analysis found that the selection signatures were related to the positive regulation of muscle cell proliferation. These results confirm the importance of Chinese Jinnan cattle as a genetic resource for beef cattle breeding and the need to preserve populations. 


\section{Funding}

This study was supported by grants from Special Fund for Agro-scientific Research in the Public Interest (201503134), National 863 Plans Projects (2011AA10030705), Science and Technology Innovation Team of Shanxi (201605D131043), Modern Agricultural Industry Technology System of Shanxi (2017-05).

\section{Authors' contributions}

YZ, DH and JC conceived of the study, carried out the molecular biological studies and drafted the manuscript. BL and GJ participated in the sequence analysis. JC and FX performed the statistical analysis. YZ, DH and XZ coordinated and helped to complete the experience. All authors read and approved the final manuscript.

\section{ACKNOWLEDGMENTS}

The authors express gratitude to Dr. Jianfeng Liu of China Agricultural University for his assistance in data analysis. The authors would like to thank Enago (www.enago.cn) for the English language review.

\section{Additional files}

Additional file 1: Table S1. Genes associated with significant integrated haplotype scores in t he Chinese Jinnan breed.

Additional file 2: Table S2. Enriched Gene Ontology terms for genes in regions under selection in Chinese Jinnan cattle.

Additional file 3: Table S3. Regions under selection in Chinese Jinnan ca ttle compared with quantitative trait loci and genome-wide association study data.

\section{REFERENCES}

Akey, J. M., G. Zhang, K. Zhang, L. Jin and M. D. Shriver. 2002. Interrogating a high-density SNP map for signatures of natural selection. Genome Res. 12: 1805-1814.

An, D., X. Dong and Z. Dong. 2005. Prokaryote diversity in the rumen of yak (Bos grunniens) and Jinnan cattle (Bos taurus) estimated by $16 \mathrm{~S}$ rDNA homology analyses. Anaerobe. 11: 207-215.

Barendse, W., B. E. Harrison, R. J. Bunch, M. B. Thomas and L.B. Turner. 2009. Genome wide signatures of positive selection: The comparison of independent samples and the identification of regions associated to traits. BMC Genomics. 10: 178.

Biswas, S. and J. M. Akey. 2006. Genomic insights into positive selection. Trends Genet. 2: 437-446.

Doran, A. G., D. P. Berry and C. J. Creevey. 2014. Whole genome association study identifies regions of the bovine genome and biological pathways involved in carcass trait performance in Holstein-Friesian cattle. BMC Genomics. 15: 837.

Fay, J.C. and C. I. Wu. 2000. Hitchhiking under positive Darwinian selection. Genetics. 155: 1405-1413.

Gautier, M. and M. Naves. 2011. Footprints of selection in the ancestral admixture of a New World Creole cattle breed. Mol Ecol. 20: 3128-3143.

Gautier, M. and R. Vitalis. 2012. Rehh: An R package to detect footprints of selection in genome-wide SNP data from haplotype structure. Bioinformatics. 28: 1176-1177.

Huang, D. W., B. T. Sherman and R. A. Lempicki. 2009. Bioinformatics enrichment tools: Paths toward the comprehensive functional analysis of large gene lists. Nucleic Acids Res. 37: 1-13.

Kasarda, R., N. Moravčíková, A. Trakovická, G. Mészáros and O. Kadlečík. 2015. Genome-wide selection signatures in Pinzgau cattle. Potravinarstvo. 9: 268-274.

Korzelius, J., I. The, S. Ruijtenberg, M. B. W. Prinsen, V. Portegijs, T. C. Middelkoop, M. J. G. Koerkamp, F. C. P. Holstege, M. Boxem and S. van den Heuvel. 2011. Caenorhabditis elegans cyclin D/CDK4 and cyclin E/CDK2 induce distinct cell cycle reentry programs in differentiated muscle cells. PLoS Genet. 7: e1002362.

Lazaro, J. B., P. J. Bailey and A. B. Lassar. 2002. Cyclin D-cdk4 activity modulates the subnuclear localization and interaction of MEF2 with SRC-family coactivators during skeletal muscle differentiation. Gene. Dev. 16: 1792-1805.

Liu, Y., H. Wang, G. Cheng, B. Jiang and L. Zan. 2014. Expression analysis of Gli1 and Gli2 in different tissues and muscle-derived cells of Qinchuan cattle. Genet. Mol. Res. 13: 8767-8775.

McClure, M. C., H. R. Ramey, M. M. Rolf, S. D. McKay, J. E. Decker, R. H. Chapple, J. W. Kim, T. M. Taxis, R. L. Weaber, R. D. Schnabel and J. F. Taylor. 2012. Genome-wide association analysis for quantitative trait loci influencing Warner-Bratzler shear force in five taurine cattle breeds. Anim. Genet. 43: 662-673.

McDonald, J. H. and M. Kreitman. 1991. Adaptive protein evolution at the Adh locus in Drosophila. Nature. 351: 652-654.

Nalaila, S. M., P. Stothard, S. S. Moore, C. Li and Z. Wang. 2012. Whole-genome QTL scan for ultrasound and carcass merit traits in beef cattle using Bayesian shrinkage method. J. Anim. Breed. Genet. 129: 107-119.

Nishimura, S., T. Watanabe, K. Mizoshita, K. Tatsuda, T. Fujita, N. Watanabe, Y. Sugimoto and A. Takasuga. 2012. Genomewide association study identified three major QTL for carcass weight including the PLAG1-CHCHD7 QTN for stature in Japanese Black cattle. BMC Genet. 13: 40.

Pan, D., S. Zhang, J. Jiang, L. Jiang, Q. Zhang and J. Liu. 2013. Genome-wide detection of selective signature in Chinese Holstein. PLoS One. 8: e60440.

Qanbari, S., D. Gianola, B. Hayes, F. Schenkel, S. Miller, S. Moore, G. Thaller and H. Simianer. 2011. Application of site and haplotype-frequency based approaches for detecting selection signatures in cattle. BMC Genomics. 12: 318.

Qanbari, S., E. C. Pimentel, J. Tetens, G. Thaller, P. Lichtner, A. R. Sharifi and H. Simianer. 2010. A genome-wide scan for signatures of recent selection in Holstein cattle. Anim. Genet. 41: 377-389.

Rocha, D., C. Billerey, F. Samson, D. Boichard and M. Boussaha. 2014. Identification of the putative ancestral allele of bovine single-nucleotide polymorphisms. J. Anim. Breed. Genet. 131: 483-486.

Saatchi, M., R. D. Schnabel, J. F. Taylor and D. J. Garrick. 2014 Large-effect pleiotropic or closely linked QTL segregate within and across ten US cattle breeds. BMC Genomics. 15: 442.

Sabeti, P. C., D. E. Reich, J. M. Higgins, H. Z. Levine, D. J. Richter, S. F. Schaffner, S. B. Gabriel, J. V. Platko, N. J. Patterson, G. J. McDonald, H. C. Ackerman, S. J. Campbell, D. Altshuler, R. Cooper, D. Kwiatkowski, R. Ward and E. S. Lander. 2002. 
Detecting recent positive selection in the human genome from haplotype structure. Nature. 419: 832-837.

Veneroni-Gouveia, G., S. L. Meirelles, D. A. Grossi, A. C. Santiago, T. S. Sonstegard, M. E. Yamagishi, L. K. Matukumalli, L. L. Coutinho, M. M. Alencar, H. N. Oliveira and L. C. Regitano. 2012. Whole-genome analysis for backfat thickness in a tropically adapted, composite cattle breed from Brazil. Anim. Genet. 43: 518-524.

Voight, B. F., S. Kudaravalli, X. Q. Wen and J. K. Pritchard. 2006a. A map of recent positive selection in the human genome. PLoS Biol. 4: e72.

Voight, B. F., S. Kudaravalli, X. Q. Wen and J. K. Pritchard. 2006b. A map of recent positive selection in the human genome. PLoS Biol. 4: 446-458.

von Baer, A., A. Ehrhardt, D. Baumhoer, R. Mayer-Steinacker, M. Schultheiss, T. Abdul-Nou, T. Mentzel, F. Fend, P. Moller, G. Jundt and T. F. E. Barth. 2014. Immunohistochemical and FISH analysis of MDM2 and CDK4 in a dedifferentiated extraskeletal osteosarcoma arising in the vastus lateralis muscle: Differential diagnosis and diagnostic algorithm. Pathol. Res. Pract. 210: 698-703.

von Grabowiecki, Y., C. Licona, L. Palamiuc, P. Abreu, V. Vidimar, D. Coowar, G. Mellitzer and C. Gaiddon. 2015. Regulation of a Notch3-Hes1 pathway and protective effect by a tocopherol-omega alkanol chain derivative in muscle atrophy. J. Pharmacol. Exp. Ther. 352: 23-32.

Willing, E. M., P. Bentzen, C. van Oosterhout, M. Hoffmann, J. Cable, F. Breden, D. Weigel and C. Dreyer. 2010. Genomewide single nucleotide polymorphisms reveal population history and adaptive divergence in wild guppies. Mol. Ecol. 19: 968-984.

Xu, L., D. M. Bickhart, J. B. Cole, S. G. Schroeder, J. Song, C. P. Tassell, T. S. Sonstegard and G. E. Liu. 2015. Genomic signatures reveal new evidences for selection of important traits in domestic cattle. Mol. Biol. Evol. 32: 711-725.

Zhao, F., S. McParland, F. Kearney, L. Du and D. P. Berry. 2015. Detection of selection signatures in dairy and beef cattle using high-density genomic information. Genet. Sel. Evol. 47: 49. 